\title{
HRTEM Study of InN Films Grown on Sapphire - Evidence for a Cubic InN Structure
}

\author{
Zongwen Liu, ${ }^{*}$ Robert J. Kinsey,** Steven M. Durbin, ${ }^{* *}$ and Simon P. Ringer* \\ * The Australian Key Centre for Microscopy and Microanalysis, The University of Sydney, NSW \\ 2006, Australia \\ ** Department of Electrical and Computer Engineering, MacDiarmid Institute for Advanced \\ Materials and Nanotechnology, University of Canterbury, Christchurch, New Zealand
}

The III-nitride semiconductors play an important role in many electronic devices such as laser diodes and high electron mobility transistors. While the band structure of GaN and AIN are relatively well understood, the band parameters of $\mathrm{InN}$ are still under debate - even the bandgap of InN is an issue and the results from different research groups are inconsistent. The historically accepted bandgap of $\mathrm{InN}$ is $1.89 \mathrm{eV}$, determined from transmission measurements on sputtered films [1]. More recent optical measurements of higher quality films suggest a band gap of $\sim 0.7 \mathrm{eV}$ [2]. Various explanations for the discrepancy have been offered, including a recent Mie resonances study by Shubina et al. which claims that optical emission from the InN films will be red shifted by interactions with indium metal particles found in the film. In this study we present high-resolution transmission electron microscopy (HRTEM) results to show no evidence of indium precipitates, however nanodomains of InN with a cubic zincblende structure have been observed in InN films grown on sapphire.

The two InN films for the present study were prepared through plasma assisted molecular beam epitaxy (PAMBE) at the University of Canterbury. Various thicknesses of low temperature GaN buffer layer were grown on the sapphire substrates, prior to growing the InN films.

HRTEM reveals that the InN layer grown on the thick GaN buffer $(\sim 400 \mathrm{~nm})$ is relatively uniform in structure and orientation. whereas the InN layer grown on the thin $\mathrm{GaN}$ buffer $(\sim 100 \mathrm{~nm})$ is a mosaic of nanodomains which are a few nm across and all rotated slightly with respect to each other (FIG 1). Although the mosaic nanodomains were also observed in the InN film grown on the thick GaN buffer, they are less common than in the InN film grown on the thin buffer. HRTEM and nanobeam electron diffraction analysis show that while both InN films have a hexagonal wurtzite structure, the mosaic nanodomains found in both films are cubic InN, with a zincblende structure.

No indium precipitates were observed in either of the InN films, eliminating the possibility of Mie resonances; however, the coexistence of cubic InN within the hexagonal structure may shed light on the large range in the observed optical properties of InN films. In an attempt to determine the properties of cubic InN we have also grown InN films on cubic (100) yttrium stabilized zirconia (YSZ). HRTEM shows that the initial film growth is cubic, but by the time it reaches a thickness of $300 \mathrm{~nm}$ it is a mix of cubic and hexagonal phases.

\section{References}

[1] T. L. Tansley and C. P. Foley, J. Appl. Phys. 59 (1986) 3241.

[2] J. Wu et al., Appl. Phys. Lett. 80, 3967 (2002).

[3] T. V. Shubina et al., Phys. Rev. Lett. 92 (2004) 117407. 


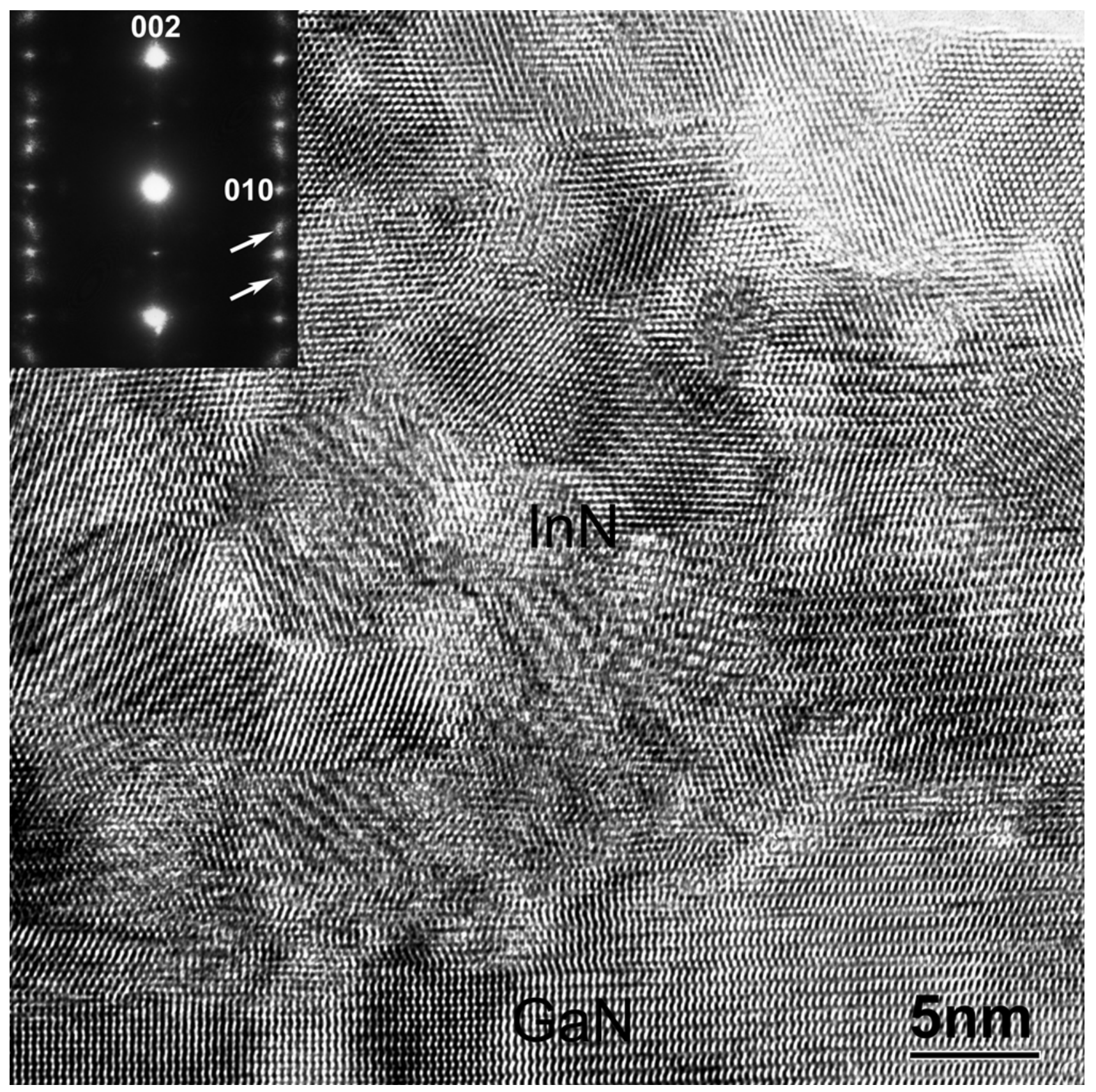

Fig. 1. HRTEM image showing InN nanodomains and GaN (thin buffer) in [100] orientation. The inset is a SAED pattern of the InN film only. Extra reflections such as the arrowed ones are from the nanodomains. 